\title{
Stochastic Multi-Objective Programming Problem: A Two-Phase Weighted Coefficient Approach
}

\author{
Hamiden Abd El-Wahed Khalifa ${ }^{1,2}$, Pavan Kumar ${ }^{3}$, Sultan S. Alodhaibi ${ }^{* *}$ \\ ${ }^{1}$ Operations Research Department, Faculty of Graduate Studies for Statistical Research, Cairo University, Giza 12613, Egypt \\ ${ }^{2}$ Department of Mathematics, College of Science and Arts, Al-Badaya, Qassim University, Buraydah 51951, Saudi Arabia \\ ${ }^{3}$ Mathematics Division, School of Advanced Science and Languages, VIT Bhopal University, Sehore 466114, MP, India \\ ${ }^{4}$ Mathematics Department, College of Science and Arts, AL-Rass, Qassim Univesity, Al- Rass 51921, Saudi Arabia
}

Corresponding Author Email: pavankmaths@ gmail.com

https://doi.org/10.18280/mmep.080603

Received: 13 May 2021

Accepted: 13 August 2021

\section{Keywords:}

chance-constrained programming, compromise index, multi-objective programming, pareto optimal solution, two-phase approach

\begin{abstract}
This paper deals with multi-objective stochastic linear programming problem. The problem is considered by introducing the coefficients of the decision variables and the right-hand-side parameters in the constraints as normal random variables. A method for converting the problem into its deterministic problem is proposed and hence two- phase approach with equal weights is proposed for finding an efficient solution. The advantages of the approach are: as weights which is positive, not necessarily equal and generate an efficient solution. A numerical example is given to illustrate the suggested methodology.
\end{abstract}

\section{INTRODUCTION}

Stochastic programming deals with the theory and methods of incorporating stochastic variations into a mathematical programming problem [1]. In most of the real life problems in mathematical programming, the parameters are considered as random variables. The branch of mathematical programming which deals with the theory and methods for the solution of conditional extremum problems under incomplete information about the random parameters is called "Stochastic programming". Most of the problems in applied mathematics may be considered as belonging to any one of the following classes [2]:

1. Descriptive Problems, in which, with the help of mathematical methods, information is processed about the investigated event some laws of the event being included by others.

2. Optimization Problems in which from a set of feasible solutions, an optimal solution is chosen.

Beside the above division of applied mathematics problems, they may be further classified as deterministic and stochastic problems. In the process of the solution of the stochastic problem, several mathematical methods have been developed. However, probabilistic methods were for a long time applied exclusively to the solution of the descriptive type of problems. Research on the theoretical development of stochastic programming is going on for the last four decades. To the several real-life problems in management science, it has been applied successfully [3]. The basic idea of all stochastic programming models is converting the probabilistic nature of the problem into an equivalent deterministic situation [4].

There are several discussions about fuzzy methods; information is approaches for solving multi-objective optimization problems. Mcadansky [5] studied the inequalities for stochastic linear programming problem. Zimmerman [6] applied the min- operator for these problems. Guu and $\mathrm{Wu}$ [7] proposed two- phase approach for solving multi-objective linear programming. While, some other authors, like Hulsurkar et al. [8], Caballero et al. [9], etc., studied the fuzzy programming methodology to solve the multi- objective stochastic linear programs. They considered the stochastic approach versus multi objective approach. Lai and $\mathrm{Ng}$ [10] studied some applications of stochastic approach to hotel revenue optimization. Stanch-Minasian [11] present a review paper on stochastic single objective linear programming. Santoso et al. [12] studied the supply chain network design with uncertainty, and proposed a stochastic programming approach.

Three approaches for stochastic programming are developed (Goicoechea et al. [13]).

Two major approaches are as follows:

(i) Chance constrained programming which can be used to solve problems having finite probability of violated by Charnes and Cooper [14];

(ii) Two-stage programming which has suggested by Dantzig and Infanger [15] and does not allow any constraints to be violated.

For several years, stochastic model has applied to deal the probabilistic uncertainty in parameters. Abbas and Bellahcene [16] introduced a cutting plane method to solve the multi objective stochastic integer linear program. Goh et al. [17] and Azaron et al. [18] investigated the stochastic model applications to the area of risk management in supply chain networks. Sakawa and Matsui [19] addressed a fuzzy solution technique to multi objective stochastic integer programming problem. They considered the simple recourse model in their proposed study. Han et al. [20] studied the interval-parameter multi-stage stochastic mixed integer programming model. They considered the probabilistic-constraints to cope with the uncertainty, and presented an application to inter-basin water 
resources management system. Körpeoğlu et al. [21] studied the production scheduling problem. They used the multi-stage stochastic programming approach to solve the scheduling problem. Birge and Louveaux [22] presented several applications of stochastic programming approach in the field of logistics, production scheduling and inventory management. Wang and Watada [23] studied the fuzzy stochastic programming with two-stages under the criteria of value-atrisk.

The researchers studied various applications of stochastic models in last few decades. Barik et al. [24] introduced the interval discrete random variables. They used these variables to solve the Multi objective stochastic program with twostages. Abdelaziz [25] and developed some solution methods to multi objective stochastic programming. Gutjahr and Pichler [26] presented a detailed survey on the methods of stochastic multi-objective optimization. Housh et al. [27] applied the stochastic programming to deal the water supply system management. Kumar and Dutta [28] studied the fuzzy approaches to inventory management systems. Kiliç and Tuzkaya [29] proposed a two-stage stochastic mixed-integer programming model. They used this model to study the physical distribution network design problem. Dutta and Kumar [30] studied the inventory management problem with time-varying demand. Yu and Solvang [31] studied a scenariobased solution method to solve the stochastic programming problem with improved multi-criteria. They presented an application to sustainable reverse logistics design of waste equipment. Khalifa et al. [32] applied fuzzy programming approach for solving multi-objective quadratic programming with all the parameters in all of objective functions and constraints are normally distributed. Yu and Solvang [33] formulated a new fuzzy- stochastic multi- objective mathematical model for sustainable closed- loop supply chain network design aims at balancing the trade- off between cost effectiveness and environmental performance under different types of uncertainty. Caglayan and Satoglu [34] used the multi- objective two- stage stochastic programming model to minimize the numbers of unserved casualties, ambulances and the total transportation time by creating scenarios based on uncertain factors.

In the past few decades, several researches presented their work on stochastic programming with various application to diverse fields of study. Mohamadi and Yaghoubi [35] presented an earthquake case study with an application of biobjective stochastic model under disruptions. They investigated the stochastic model for emergency medical services network design. The assumed the backup services for disasters. Restrepo et al. [36] and Floyd et al. [37] studied the tour scheduling problem and project management with an application of two stage stochastic programming technique. Farrokh et al. [38] studied the closed loop supply chain network design under hybrid uncertainty with the use of fuzzy stochastic programming. Rahmanniyay and $\mathrm{Yu}$ [39] introduced a new multi-objective mathematical model in which objective functions optimize cost and competency simultaneously to develop a project team for multidisciplinary projects under uncertainty.

An integrated chance-constrained stochastic model was proposed to supply chain network problem for a mobile phone by Ahmadi and Amin [40]. Khalifa and Kumar [41] presented a multi-objective optimization problem to solve the cooperative continuous static games. They used the KarushKuhn-Tucker conditions. Waliv et al. [42] studied the stochastic multi objective inventory model with uncertain nature of parameters. They used the nonlinear programming approach to solve the stochastic problem. Yu and $\mathrm{Li}$ [43] formulated the logistic problem as a stochastic problem. They used the robust optimization model to solve the stochastic problem. Yenice and Samanlioglu [44] studied the earthquake relief network problem with applications to multi-objective stochastic approach. Very recently, Khalifa et al. [45] studied the multi objective programming in fuzzy environment with an application to transportation problem.

In this paper, stochastic multi-objective programming problem is considered, where the right and left-hand side values of the constraints are random variables with known distribution. Firstly, the problem is converted into an equivalent deterministic form and then solved using the twophase weighted coefficients approach.

The remainder of the paper is organized as follows: In Section 2, stochastic multi-objective programming problem is formulated. Section 3 introduced solution procedure for obtaining a pareto optimal solution. Section 4 introduces some of basic results related to the problem solution. In Section 5, numerical example is introduced for illustrate the methodology. Finally, some concluding remarks are reported in Section 6.

\section{PROBLEM FORMULATION AND SOLUTION CONCEPTS}

A multi-objective stochastic programming with some chance- constrains can be stated as:

$$
\operatorname{Max} \mathrm{Z}_{\mathrm{k}}(\mathrm{x})=\sum_{\mathrm{j}=1}^{\mathrm{n}} \mathrm{c}_{\mathrm{j}}^{(\mathrm{k})} \mathrm{x}_{\mathrm{j}}, \mathrm{k}=1,2, \ldots, \mathrm{K}
$$

Subject to

$$
\begin{gathered}
P\left(\sum_{j=1}^{n} a_{i j} x_{j} \leq b_{i}\right) \geq 1-\gamma_{i}, i=\overline{1, m}, \\
x_{j}, j=\overline{1, n}, 0<\gamma_{i}<1, i=\overline{1, m},
\end{gathered}
$$

where, $\mathrm{Z}_{\mathrm{k}}=\left(\mathrm{Z}_{1}, \mathrm{Z}_{2}, \ldots, \mathrm{Z}_{\mathrm{K}}\right), \mathrm{C}$ is the cost coefficient matrix, $x$ is the decision vector, $A=\left(\mathrm{a}_{\mathrm{ij}}\right)_{\mathrm{m} \times \mathrm{n}}$ is the coefficient matrix, and $b$ is the right hand side vector, $\mathrm{a}_{\mathrm{ij}}$ and $\mathrm{b}_{\mathrm{i}}$ are random normal variables and $0<\gamma_{\mathrm{i}}<1$ are specific probabilities. Eq. (2) indicates that the $i^{\text {th }}$ constraints $\sum_{\mathrm{j}=1}^{\mathrm{n}} \mathrm{a}_{\mathrm{ij}} \mathrm{x}_{\mathrm{j}} \leq \mathrm{b}_{\mathrm{i}}$ has to be satisfied with a probability of at least $\left(1-\gamma_{i}\right)$, where $0<$ $\gamma_{i}<1$. Let us consider the problem with $a_{i j}$ and $b_{i}$ are normally distributed with known means and variances.

\subsection{When $a_{i j}$ are only random variables}

Let the mean and variance of $\mathrm{a}_{\mathrm{ij}}$ denoted by $\mu\left(\mathrm{a}_{\mathrm{ij}}\right)$ and $\mathrm{V}\left(\mathrm{a}_{\mathrm{ij}}\right)$, respectively. Also, assume that the covariance between $\mathrm{a}_{\mathrm{ij}}$ and $\mathrm{a}_{\mathrm{rl}}$ is known. Now, let us define:

$$
e_{i}=\sum_{j=1}^{n} a_{i j} \leq b_{i}, i=\overline{1, m}
$$


Then, we obtain

$$
\begin{gathered}
\mu\left(e_{i}\right)=\mu\left(\sum_{j=1}^{n} a_{i j} x_{j} \leq b_{i}\right)=\sum_{j=1}^{n} \mu\left(a_{i j} x_{j}\right) \leq b_{i}, i \\
=\overline{1, m}
\end{gathered}
$$

Also,

$$
V\left(e_{i}\right)=X^{T} \sigma_{i j, r l}^{2} X, i=\overline{1, m} .
$$

Here, $\sigma_{\mathrm{ij}, \mathrm{rl}}^{2}$ is the covariance matrix, which is defined as follows:

$$
\sigma_{\mathrm{i} 1, \mathrm{i} 2}^{2}=\left(\begin{array}{ccc}
V\left(\mathrm{a}_{\mathrm{i} 1}\right) & V\left(\mathrm{a}_{\mathrm{i} 1}, \mathrm{a}_{\mathrm{i} 2}\right) & \ldots \mathrm{V}\left(\mathrm{a}_{\mathrm{i} 1}, \mathrm{a}_{\mathrm{in}}\right) \\
\mathrm{V}\left(\mathrm{a}_{\mathrm{i} 2}, \mathrm{a}_{\mathrm{i} 1}\right) & \mathrm{V}\left(\mathrm{a}_{\mathrm{i} 2}\right) \ldots & \mathrm{V}\left(\mathrm{a}_{\mathrm{i} 2}, \mathrm{a}_{\mathrm{in}}\right) \\
\ldots & \ldots & \ldots \\
V\left(\mathrm{a}_{\mathrm{in}}, \mathrm{a}_{\mathrm{i} 1}\right) & \mathrm{V}\left(\mathrm{a}_{\mathrm{in}}, \mathrm{a}_{\mathrm{i} 2}\right) & \ldots \mathrm{V}\left(\mathrm{a}_{\mathrm{in}}\right)
\end{array}\right)
$$

Hence, the constraints in (2) becomes as follows:

$$
\begin{gathered}
P\left(e_{i} \leq b_{i}\right) \geq 1-\delta_{i}, \text { i.e., } \\
P\left(\frac{e_{i}-\mu\left(e_{i}\right)}{\sqrt{V\left(e_{i}\right)}} \leq \frac{b_{i}-\mu\left(b_{i}\right)}{\sqrt{V\left(b_{i}\right)}}\right) \geq 1-\delta_{i}, i=\overline{1, m}
\end{gathered}
$$

It is observed that $\frac{e_{i}-\mu\left(e_{i}\right)}{\sqrt{V\left(e_{i}\right)}}$ is the standard normal variables. In view of this, inequality (7) can be rewritten as:

$$
\mathrm{P}\left(\mathrm{e}_{\mathrm{i}} \leq \mathrm{b}_{\mathrm{i}}\right)=\phi\left(\frac{\mathrm{b}_{\mathrm{i}}-\mu\left(\mathrm{b}_{\mathrm{i}}\right)}{\sqrt{\mathrm{V}\left(\mathrm{b}_{\mathrm{i}}\right)}}\right)
$$

Here, $\phi(z)$ in the cumulative density function of the standard normal variables at $\mathrm{z}$.

Also,

$$
\phi\left(\Psi_{\delta_{\mathrm{i}}}\right)=1-\delta_{\mathrm{i}},
$$

where, $\Psi_{\delta_{\mathrm{i}}}$ is standard normal variable value, and by referring to the inequality (6), we obtain,

$$
\phi\left(\frac{\mathrm{b}_{\mathrm{i}}-\mu\left(\mathrm{b}_{\mathrm{i}}\right)}{\sqrt{\mathrm{V}\left(\mathrm{b}_{\mathrm{i}}\right)}}\right) \geq \phi\left(\Psi_{\delta_{\mathrm{i}}}\right), \mathrm{i}=\overline{1, \mathrm{~m}}
$$

Inequality (10) is satisfied only if,

$$
\frac{\mathrm{b}_{\mathrm{i}}-\mu\left(\mathrm{b}_{\mathrm{i}}\right)}{\sqrt{\mathrm{V}\left(\mathrm{b}_{\mathrm{i}}\right)}} \geq \Psi_{\delta_{\mathrm{i}}},
$$

which can be written as follows:

$$
\mu\left(b_{i}\right)+\Psi_{\delta_{i}} \sqrt{V\left(b_{i}\right)} \leq b_{i}, i=\overline{1, m}
$$

From (5), (6) and (12), we have:

$$
\mu\left(\sum_{j=1}^{n} a_{i j} x_{j} \leq b_{i}\right)+\Psi_{\delta_{i}} \sqrt{X^{T} \sigma_{i j, r l}^{2} X} \leq b_{i}, i=\overline{1, m}
$$

Thus, problem (1)-(3) become

$$
\begin{gathered}
\operatorname{Max}_{\mathrm{k}}(\mathrm{x})=\sum_{\mathrm{j}=1}^{\mathrm{n}} \mathrm{c}_{\mathrm{j}}^{(\mathrm{k})} \mathrm{x}_{\mathrm{j}}, \mathrm{k}=1,2, \ldots, \mathrm{K} \text { Subject } \\
\operatorname{to} \mu\left(\sum_{\mathrm{j}=1}^{\mathrm{n}} \mathrm{a}_{\mathrm{ij}} \mathrm{x}_{\mathrm{j}} \leq \mathrm{b}_{\mathrm{i}}\right)+\Psi_{\delta_{\mathrm{i}}} \sqrt{\mathrm{X}^{\mathrm{T}} \sigma_{\mathrm{ij}, \mathrm{rl}}^{2} \mathrm{X}} \leq \mathrm{b}_{\mathrm{i}}, \mathrm{i}= \\
\overline{1, \mathrm{~m}}, \mathrm{x}_{\mathrm{j}}, \mathrm{j}=\overline{1, \mathrm{~m}}, 0<\delta_{\mathrm{i}}<1 .
\end{gathered}
$$

Remark 1. The covariance will be vanished if all $\mathrm{a}_{\mathrm{ij}}$ are independent.

\subsection{When $b_{i}$ are only random variables}

Let the mean and variance of $b_{i}$ are denoted by $\mu\left(b_{i}\right)$ and $V\left(\mathrm{~b}_{\mathrm{i}}\right)$; respectively. Then, constraints (3) can be rewritten as:

$$
\begin{aligned}
P\left(\frac{b_{i}-\mu\left(b_{i}\right)}{\sqrt{V\left(b_{i}\right)}}\right) & \geq \frac{\sum_{j=1}^{n} a_{i j} x_{j}-\mu\left(b_{i}\right)}{\sqrt{V\left(b_{i}\right)}} \geq 1-\delta_{i}, i \\
& =\frac{V}{1, m}
\end{aligned}
$$

Inequality (15) can be expressed as follows:

$$
\begin{aligned}
\mathrm{P}\left(\frac{\mathrm{b}_{\mathrm{i}}-\mu\left(\mathrm{b}_{\mathrm{i}}\right)}{\sqrt{\mathrm{V}\left(\mathrm{b}_{\mathrm{i}}\right)}}\right) & \leq \frac{\sum_{\mathrm{j}=1}^{\mathrm{n}} \mathrm{a}_{\mathrm{ij}} \mathrm{x}_{\mathrm{j}}-\mu\left(\mathrm{b}_{\mathrm{i}}\right)}{\sqrt{\mathrm{V}\left(\mathrm{b}_{\mathrm{i}}\right)}} \leq 1-\delta_{\mathrm{i}}, \mathrm{i} \\
& =\frac{1, \mathrm{~m}}{}
\end{aligned}
$$

In the case of $\phi\left(\Psi_{\delta_{\mathrm{i}}}\right)$ is the standard normal variable value at $\phi\left(\Psi_{\delta_{\mathrm{i}}}\right)=1-\delta_{\mathrm{i}}, \mathrm{i}=\overline{1, \mathrm{~m}}$, then inequality (16) can be rewritten as:

$$
\phi\left(\frac{\sum_{\mathrm{j}=1}^{\mathrm{n}} \mathrm{a}_{\mathrm{ij}} \mathrm{x}_{\mathrm{j}}-\mu\left(\mathrm{b}_{\mathrm{i}}\right)}{\sqrt{\mathrm{V}\left(\mathrm{b}_{\mathrm{i}}\right)}}\right) \leq \phi\left(\Psi_{\delta_{\mathrm{i}}}\right)
$$

Inequality (17) can be satisfied if:

$$
\begin{gathered}
\frac{\sum_{j=1}^{n} a_{i j} x_{j}-\mu\left(b_{i}\right)}{\sqrt{V\left(b_{i}\right)}} \leq \Psi_{\delta_{i}}, i=\overline{1, m} \quad \text { i.e., } \\
\sum_{j=1}^{n} a_{i j} x_{j} \leq \mu\left(b_{i}\right)+\Psi_{\delta_{i}} \sqrt{V\left(b_{i}\right)}, \quad i=\overline{1, m}
\end{gathered}
$$

Thus, problem (1)- (3) is equivalent to the following deterministic problem:

$$
\begin{gathered}
\operatorname{Max} Z_{k}(x)=\sum_{j=1}^{n} c_{j}^{(k)} x_{j}, k=1,2, \ldots, K \quad \text { Subject to } \\
\sum_{j=1}^{n} a_{i j} x_{j} \leq \mu\left(b_{i}\right)+\Psi_{\delta_{i}} \sqrt{V\left(b_{i}\right)}, i=\overline{1, m} \\
x_{j} \geq 0 ; \forall j .
\end{gathered}
$$

Using min-operator proposed by Zimmermann [6], problem (20) can be rewritten as follows:

$$
\begin{gathered}
\text { Max } \theta \quad \text { Subject to } \\
\theta \leq \frac{\mathrm{Z}_{\mathrm{k}}(\mathrm{x})-\mathrm{Z}_{\mathrm{k}}^{\mathrm{L}}}{\mathrm{Z}_{\mathrm{k}}^{\mathrm{U}}-\mathrm{Z}_{\mathrm{k}}^{\mathrm{L}}}, k=1,2, \ldots, K, \\
\sum_{\mathrm{j}=1}^{\mathrm{n}} \mathrm{a}_{\mathrm{ij}} \mathrm{x}_{\mathrm{j}} \leq \mu\left(\mathrm{b}_{\mathrm{i}}\right)+\Psi_{\delta_{\mathrm{i}}} \sqrt{\mathrm{V( \textrm {b } _ { \mathrm { i } } )}}, \mathrm{i}=\overline{1, \mathrm{~m}},
\end{gathered}
$$


where, $\mathrm{Z}_{\mathrm{k}}^{\mathrm{L}}$ and $\mathrm{Z}_{\mathrm{k}}^{\mathrm{U}}$ are the lower and upper bounds for each objective functions.

Let us assume that the membership function for each objective of problem (20) is equally important. Using the average operator, problem (2) can be viewed as:

$$
\begin{gathered}
\operatorname{Max} \bar{\theta}=\frac{1}{K} \sum_{k=1}^{K} \theta_{k} \quad \text { Subject to } \\
\theta_{k} \leq \frac{\mathrm{Z}_{\mathrm{k}}(\mathrm{x})-\mathrm{Z}_{\mathrm{k}}^{\mathrm{L}}}{\mathrm{Z}_{\mathrm{k}}^{\mathrm{U}}-\mathrm{Z}_{\mathrm{k}}^{\mathrm{L}}}, k=1,2, \ldots, K, \\
\sum_{\mathrm{j}=1}^{\mathrm{n}} \mathrm{a}_{\mathrm{ij}} \mathrm{x}_{\mathrm{j}} \leq \mu\left(\mathrm{b}_{\mathrm{i}}\right)+\Psi_{\delta_{\mathrm{i}}} \sqrt{\mathrm{V}\left(\mathrm{b}_{\mathrm{i}}\right)}, \mathrm{i}=\overline{1, \mathrm{~m}}, \\
\mathrm{x}_{\mathrm{j}} \geq 0 ; \forall j .
\end{gathered}
$$

The two-phase approach is the combination of the minimum operator and the average operator. So, problem (20) with the two-phase approach becomes as presented in problem (23) below:

$$
\begin{gathered}
\operatorname{Max} \hat{\theta}=\frac{1}{K} \sum_{k=1}^{K} \theta_{k} \quad \text { Subject to } \\
\theta^{*} \leq \theta_{k} \leq \frac{\mathrm{Z}_{\mathrm{k}}(\mathrm{x})-\mathrm{Z}_{\mathrm{k}}^{\mathrm{L}}}{\mathrm{Z}_{\mathrm{k}}^{\mathrm{U}}-\mathrm{Z}_{\mathrm{k}}^{\mathrm{L}}}, k=1,2, \ldots, K, \\
\sum_{\mathrm{j}=1}^{\mathrm{n}} \mathrm{a}_{\mathrm{ij}} \mathrm{x}_{\mathrm{j}} \leq \mu\left(\mathrm{b}_{\mathrm{i}}\right)+\Psi_{\delta_{\mathrm{i}}} \sqrt{\mathrm{V}\left(\mathrm{b}_{\mathrm{i}}\right)}, \mathrm{i}=\overline{1, \mathrm{~m}}, \\
\mathrm{x}_{\mathrm{j}} \geq 0 ; \forall j .
\end{gathered}
$$

\section{BASIC RESULTS}

Definition 1. (Guu and $\mathrm{Wu}$ [7]). Let $x^{\circ}$ and $x^{*}$ be two feasible solutions of problem (20). $x^{*}$ is more efficient than $x^{\circ}$ which is denoted by if $\left(x^{*}>x^{\circ}\right), \mathrm{Z}_{\mathrm{k}}\left(x^{*}\right) \geq \mathrm{Z}_{\mathrm{k}}\left(x^{\circ}\right) ; \forall \mathrm{k}$ and $\mathrm{Z}_{\mathrm{q}}\left(x^{*}\right)>\mathrm{Z}_{\mathrm{q}}\left(x^{\circ}\right)$, for some $\mathrm{q}$.

Definition 2. (Guu and $\mathrm{Wu}$ [7]). For a feasible solution $x^{*}$, $x^{*}$ is said to be a pareto optimal solution if there does not exist a $x^{\circ}$ such that $x^{\circ}>x^{*}$.

For a given $\epsilon_{k}$, let us consider:

$$
\begin{gathered}
\operatorname{Max} \theta^{x^{*}}=\frac{1}{K} \sum_{k=1}^{K} \epsilon_{k} \theta_{k} \quad \text { Subject to } \\
\theta^{*} \leq \theta_{k} \leq \frac{\mathrm{Z}_{\mathrm{k}}(\mathrm{x})-\mathrm{Z}_{\mathrm{k}}^{\mathrm{L}}}{\mathrm{Z}_{\mathrm{k}}^{\mathrm{U}}-\mathrm{Z}_{\mathrm{k}}^{\mathrm{L}}}, k=1,2, \ldots, K, \\
\sum_{\mathrm{j}=1}^{\mathrm{n}} \mathrm{a}_{\mathrm{ij}} \mathrm{x}_{\mathrm{j}} \leq \mu\left(\mathrm{b}_{\mathrm{i}}\right)+\Psi_{\delta_{\mathrm{i}}} \sqrt{\mathrm{V}\left(\mathrm{b}_{\mathrm{i}}\right)}, \mathrm{i}=\overline{1, \mathrm{~m}}, \\
\mathrm{x}_{\mathrm{j}} \geq 0 ; \forall j .
\end{gathered}
$$

Theorem 1. If $\left(x^{*}, \theta^{x^{*}}\right)$ is an optimal solution of problem (24), then $x^{*}$ is a pareto optimal solution for problem (20).

Proof. Suppose that there exists a solution $\left(x^{*}, \theta^{x^{*}}\right)$ such that $x^{\circ}>x^{*}$. This means that

$\mathrm{Z}_{\mathrm{k}}\left(x^{*}\right) \geq \mathrm{Z}_{\mathrm{k}}\left(x^{\circ}\right) ; \forall \mathrm{k}$ and $\mathrm{Z}_{\mathrm{q}}\left(x^{*}\right)>\mathrm{Z}_{\mathrm{q}}\left(x^{\circ}\right)$, for some $\mathrm{q}$ (Definition 1). If $\mathrm{Z}_{\mathrm{q}}\left(x^{*}\right)>\mathrm{Z}_{\mathrm{q}}\left(x^{\circ}\right)$, for some $\mathrm{q}$. Then,

$$
\begin{gathered}
\theta^{*} \leq \theta_{k}^{x^{*}} \leq \frac{\mathrm{Z}_{\mathrm{k}}\left(x^{*}\right)-\mathrm{Z}_{\mathrm{k}}^{\mathrm{L}}}{\mathrm{Z}_{\mathrm{k}}^{\mathrm{U}}-\mathrm{Z}_{\mathrm{k}}^{\mathrm{L}}} \leq \frac{\mathrm{Z}_{\mathrm{k}}\left(x^{\circ}\right)-\mathrm{Z}_{\mathrm{k}}^{\mathrm{L}}}{\mathrm{Z}_{\mathrm{k}}^{\mathrm{U}}-\mathrm{Z}_{\mathrm{k}}^{\mathrm{L}}} ; \forall k \\
=1,2, \ldots, K,
\end{gathered}
$$

$\theta^{*} \leq \theta_{\mathrm{q}}^{\mathrm{x}^{*}} \leq \frac{\mathrm{Z}_{\mathrm{q}}\left(\mathrm{x}^{*}\right)-\mathrm{Z}_{\mathrm{k}}^{\mathrm{L}}}{\mathrm{z}_{\mathrm{k}}^{\mathrm{U}}-\mathrm{Z}_{\mathrm{k}}^{\mathrm{L}}} \leq \frac{\mathrm{z}_{\mathrm{q}}\left(\mathrm{x}^{\circ}\right)-\mathrm{z}_{\mathrm{k}}^{\mathrm{L}}}{\mathrm{z}_{\mathrm{k}}^{\mathrm{U}}-\mathrm{Z}_{\mathrm{k}}^{\mathrm{L}}} ;$ for some $\mathrm{q}$.

Since,

$\theta_{\mathrm{q}}^{\mathrm{x}^{*}} \leq \frac{\mathrm{z}_{\mathrm{q}}\left(\mathrm{x}^{*}\right)-\mathrm{z}_{\mathrm{k}}^{\mathrm{L}}}{\mathrm{z}_{\mathrm{k}}^{\mathrm{U}}-\mathrm{Z}_{\mathrm{k}}^{\mathrm{L}}} \leq \theta_{\mathrm{q}}^{\mathrm{x}^{\circ}} \leq \frac{\mathrm{z}_{\mathrm{q}}\left(\mathrm{x}^{\circ}\right)-\mathrm{z}_{\mathrm{k}}^{\mathrm{L}}}{\mathrm{z}_{\mathrm{k}}^{\mathrm{U}}-\mathrm{z}_{\mathrm{k}}^{\mathrm{L}}} ;$ for some $\mathrm{q}$. Then, $\sum_{\mathrm{k}=1}^{\mathrm{K}} \epsilon_{\mathrm{k}} \theta_{\mathrm{k}}=\sum_{\mathrm{i}=1, \mathrm{i} \neq \mathrm{j}}^{\mathrm{K}} \epsilon_{\mathrm{i}} \theta_{\mathrm{i}}^{\mathrm{x}^{*}}+\epsilon_{\mathrm{j}} \theta_{\mathrm{j}}^{\mathrm{x}^{*}}<\sum_{\mathrm{i}=1, \mathrm{i} \neq \mathrm{j}}^{\mathrm{K}} \epsilon_{\mathrm{i}} \theta_{\mathrm{i}}^{\mathrm{x}^{*}}+\epsilon_{\mathrm{j}} \theta_{\mathrm{j}}^{\mathrm{x}^{\circ}}$.

Thus $\left(x^{*}, \theta^{x^{*}}\right)$ is not an optimal solution to problem (24), which is contradiction.

\section{SOLUTION PROCEDURE}

In this Section, a method for solving a multi-objective stochastic programming problem is developed by using the two- phase approach. We summarized the above developed method as a solution procedure, which provides the step-bystep procedure to solve a multi-objective stochastic programming problem.

The following steps are needed to solve the proposed stochastic model by developed methodology:

Step 1: Transform the problems (1)-(3) into its deterministic version as represented by problem (20). Using the chance constrained programming.

Step 2: Solve problem (20) to determine the individual solutions.

Step 3: Determine the lower and upper bounds $\left(\mathrm{Z}_{\mathrm{k}}^{\mathrm{L}}\right.$ and $\left.\mathrm{Z}_{\mathrm{k}}^{\mathrm{U}}, k=1,2, \ldots, K\right)$.

Step 4: Construct the membership functions for fuzzy parameters.

Step 5: Formulate the problems (21), (22), and (23).

Step 6: Solve each of problems (21), (22), and (23) using any optimization software package such as Lingo, MATLAB, Mathematica, etc. Here, we preferred to use MATLAB 2020a.

\section{NUMERICAL EXAMPLE}

Consider the following stochastic multi-objective programming problem:

$$
\begin{gathered}
\operatorname{Max} Z_{1}=5 x+6 y+3 z \\
\operatorname{Max} Z_{2}=7 x+2 y+4 z \\
\operatorname{Max} z_{3}=2 x+3 y+8 z \\
\text { Subject to } \\
\mathrm{P}\left(\mathrm{a}_{11} \mathrm{x}+\mathrm{a}_{12} \mathrm{y}+\mathrm{a}_{13} \mathrm{z} \leq 8\right) \geq 0.95, \\
\mathrm{P}\left(5 \mathrm{x}+\mathrm{y}+6 \mathrm{z} \leq \mathrm{b}_{2}\right) \geq 0.1, \\
x, y, z \geq 0,
\end{gathered}
$$

where, $a_{i j}$ and $b_{2}$ are normally distributed random variables with the parameter values as presented below:

$$
\begin{gathered}
\mu\left(\mathrm{a}_{11}\right)=1, \\
\mu\left(\mathrm{a}_{12}\right)=3, \\
\mu\left(\mathrm{a}_{13}\right)=9, \\
V\left(\mathrm{a}_{11}\right)=25, \\
V\left(\mathrm{a}_{12}\right)=16, \\
V\left(\mathrm{a}_{13}\right)=4, \\
\mu\left(\mathrm{b}_{2}\right)=7, \\
V\left(\mathrm{~b}_{2}\right)=9 .
\end{gathered}
$$

and 
Step 1: The deterministic problem for problem (25) is formulated as follows:

$$
\begin{gathered}
\operatorname{Max} Z_{1}=5 x+6 y+3 z \\
\operatorname{Max} Z_{2}=7 x+2 y+4 z \\
\operatorname{Max} Z_{3}=2 x+3 y+8 z \\
\text { Subject to } \\
\mathrm{x}+3 \mathrm{y}+9 \mathrm{z} \leq 8+1.645 \mathrm{u} \leq 8 \\
25 \mathrm{x}^{2}+16 \mathrm{y}^{2}+4 \mathrm{z}^{3}-\mathrm{u}^{2}=0, \\
5 \mathrm{x}+\mathrm{y}+6 \mathrm{z} \leq 10.855 \\
\mathrm{x}, \mathrm{y}, \mathrm{z}, \mathrm{u} \geq 0 .
\end{gathered}
$$

Step 2, 3: The individual maximum solutions are illustrated in Table 1, below.

Table 1. The individual maximum solutions

\begin{tabular}{ccccc}
\hline$x$ & $y$ & $z$ & $u$ & $Z_{K}^{U}$ \\
\hline 0.4625 & 0.6327 & 0 & 3.4282 & 6.1087 \\
0.8672 & 0 & 0 & 4.3360 & 6.0705 \\
0.05976 & 0.07558 & 0.6502 & 1.1315 & 5.5481 \\
\hline
\end{tabular}

The individual minimum is: $\mathrm{Z}_{\mathrm{K}}^{\mathrm{L}}=0$, for $\mathrm{k}=1,2,3$.

Step 4: Solving problem (26) by min- operator.

$$
\begin{gathered}
\operatorname{Max} \theta \quad \text { Subject to } \\
5 x+6 y+3 z-6.1087 \theta \geq 0 \\
7 x+2 y+4 z-6.0705 \theta \geq 0 \\
2 x+3 y+8 z-5.5481 \theta \geq 0 \\
x+3 y+9 z \leq 8+1.645 u \leq 8 \\
25 x^{2}+16 y^{2}+4 z^{3}-u^{2}=0 \\
5 x+y+6 z \leq 10.855 \\
x, y, z, u \geq 0, \text { and } \theta \in[0,1] .
\end{gathered}
$$

The optimal compromise solution is as follows:

$$
\begin{gathered}
\theta^{*}=0.652126, \\
\mathrm{x}^{*}=0.468 \\
\mathrm{y}^{*}=0.264, \\
\mathrm{z}^{*}=0.270, \\
\mathrm{u}=2.623 \\
\mathrm{Z}_{1}^{*}=4.731, \\
\mathrm{Z}_{2}^{*}=4.883, \\
\mathrm{Z}_{3}^{*}=3.883
\end{gathered}
$$

The solution by average operator:

$$
\begin{gathered}
\operatorname{Max} \bar{\theta}=\frac{1}{3}\left(\theta_{1}+\theta_{2}+\theta_{3}\right) \quad \text { Subject to } \\
5 x+6 y+3 z-6.1087 \theta_{1} \geq 0 \\
7 x+2 y+4 z-6.0705 \theta_{2} \geq 0 \\
2 x+3 y+8 z-5.5481 \theta_{3} \geq 0 \\
x+3 y+9 z \leq 8+1.645 u \leq 8 \\
25 x^{2}+16 y^{2}+4 z^{3}-u^{2}=0 \\
5 x+y+6 z \leq 10.855 \\
x, y, z, u \geq 0, \theta_{1}, \theta_{2}, \theta_{3} \in[0,1]
\end{gathered}
$$

The solution is as follows:

$$
\begin{gathered}
\theta^{*}=0.629, \\
\theta_{1}=1, \\
\theta_{2}=1, \\
\theta_{3}=0, \\
\mathrm{x}^{*}=0.146, \\
\mathrm{y}^{*}=0.000001614735,
\end{gathered}
$$

$$
\begin{gathered}
\mathrm{Z}_{1}^{*}=4.731, \\
\mathrm{Z}_{2}^{*}=4.883, \\
\mathrm{Z}_{3}^{*}=3.883, \\
\mathrm{u}=0,
\end{gathered}
$$

Now, let us take $\epsilon_{1}=0$, and consider the two- phase method, and then we obtain:

$$
\begin{gathered}
\operatorname{Max} \hat{\theta}=0.5 \theta_{2}+0.5 \theta_{3} \quad \text { Subject to } \\
0.002416188 \leq \theta_{1} \leq \frac{1}{6.1087}(5 x+6 y+3 z) \\
0.002416188 \leq \theta_{2} \leq \frac{1}{6.0705}(7 x+2 y+4 z) \\
0.002416188 \leq \theta_{3} \leq \frac{1}{5.5481}(52 x+3 y+8 z) \\
x+3 y+9 z \leq 8+1.645 u \leq 8 \\
25 x^{2}+16 y^{2}+4 z^{3}-u^{2}=0 \\
5 x+y+6 z \leq 10.855 \\
x, y, z, u \geq 0, \theta_{1}, \theta_{2}, \theta_{3} \in[0,1]
\end{gathered}
$$

The solution of problem (29) is as follows:

$$
\begin{gathered}
\hat{\theta}=0.589, \\
\mathrm{x}^{*}=0 \\
\mathrm{y}^{*}=0 \\
\mathrm{z}^{*}=0.005088730, \\
\theta_{1}=0.559 \\
\theta_{2}=0.813 \\
\theta_{3}=0.537 \\
\mathrm{Z}_{1}^{*}=4.731 \\
\mathrm{Z}_{2}^{*}=4.883 \\
\mathrm{Z}_{3}^{*}=3.883
\end{gathered}
$$

It is observed that the solution resulted from the proposed approach is the same as given by Hulsurkar et al. [8]

After solving the problem using the two-phase approach, the sensitivity analysis on $\theta$ is as follows:

$$
\begin{gathered}
\mathrm{Z}_{1}^{*}=4.731-\theta^{*}, \\
\mathrm{Z}_{2}^{*}=4.883-3.883 \theta^{*}, \\
\mathrm{Z}_{3}^{*}=3.883+4.731 \theta^{*}, \\
\theta_{1}=0.629-0.081 \theta^{*}, \\
\theta_{2}=1-0.88 \theta^{*}, \\
\theta_{3}=1-0.375 \theta^{*}, \\
0 \leq \theta^{*} \leq 0.5 .
\end{gathered}
$$

Obviously, the degree of satisfaction of each individual can be represented by his/ her membership function and the highest degree is $\theta^{*}=0.5$.

\section{CONCLUSIONS}

Studying stochastic multiobjective optimization due to its close connection with human life has considered to be great important. In this paper, we have shown that by applying the two- phase approach with equal weights and positively achieved an efficient solution for the stochastic multiobjective programming problem. The same has been illustrated through a numerical example. It is quite evident that these results more benefits to the decision maker who need to allocate the resources efficiently, and who needs to treat each of them with equal weights. It has shown that the two- phase technique having positive weighted coefficients, not 
necessarily equal to solve multi-objective programming problem gives an efficient solution. For the further research, one may elaborate the multi-objective optimization in linguistic environment by characterizing with fuzzy random numbers. These results provide contributions for planner try to allocate resources efficiently. The planner needs to take care of each interest individually not treating each individual with equally importance.

\section{ACKNOWLEDGMENT}

The authors gratefully thank the Editor-in-Chief and the anonymous referees for their helpful and suggestive comments.

\section{REFERENCES}

[1] Sengupta, J., Serman, I.Z. (1973). Stochastic programming: Methods and applications. Journal of the Royal Statistical Society: Series A (General), 136(3): 460-461. https://doi.org/10.2307/2345008

[2] Sengupta, J.K. (1985). Stochastic optimization: Examples and applications. In: Information and Efficiency in Economic Decision. Advanced Studies in Theoretical and Applied Econometrics, vol 4. Springer, Dordrecht. https://doi.org/10.1007/978-94-009-50535 -1

[3] Sinha, S.B., Hulsurkar, S., Biswal, M. (2000). Fuzzy programming approach to multi-objective stochastic programming problems when bi's follow joint normal distribution. Fuzzy Sets and Systems, 109(1): 91-96. https://doi.org/10.1016/S0165-0114(98)00070-0

[4] Sinha, S.B., Biswal, M.P., Hulsurkar, S. (1998). Fuzzy programming approach to the multi-objective probabilistic linear programming problems when only $b$ i's are probabilistic. Journal of Fuzzy Mathematics, 6(1): 63-74.

[5] Madansky, A. (1960). Inequalities for stochastic linear programming problems. Management Science, 6(2): 197-204. https://doi.org/10.1287/mnsc.6.2.197

[6] Zimmermann, H.J. (1978). Fuzzy programming and linear programming with several objective functions. Fuzzy Sets and Systems, 1(1): 45-55. https://doi.org/10.1016/0165-0114(78)90031-3

[7] Guu, S.M., Wu, Y.K. (1997). Weighted coefficients in two-phase approach for solving the multiple objective programming problems. Fuzzy sets and Systems, 85(1): 45-48. ttps://doi.org/10.1016/0165-0114(95)00360-6

[8] Hulsurkar, S., Biswal, M.P., Sinha, S.B. (1997). Fuzzy programming approach to multi-objective stochastic linear programming problems. Fuzzy Sets and Systems, 88(2): $\quad 173-181 . \quad$ https://doi.org/10.1016/S01650114(96)00056-5

[9] Caballero, R., Cerdá, E., del Mar Muñoz, M., Rey, L. (2004). Stochastic approach versus multiobjective approach for obtaining efficient solutions in stochastic multiobjective programming problems. European Journal of Operational Research, 158(3): 633-648. https://doi.org/10.1016/S0377-2217(03)00371-0

[10] Lai, K.K., Ng, W.L. (2005). A stochastic approach to hotel revenue optimization. Computers \& Operations Research, 32(5): https://doi.org/10.1016/j.cor.2003.09.012
[11] Stanch-Minasian, I.M. (1976). A research bibliography in stochastic programming. Oper. Res., 24: 1078-1119. https://doi.org/10.1287/opre.24.6.1078

[12] Santoso, T., Ahmed, S., Goetschalckx, M., Shapiro, A. (2005). A stochastic programming approach for supply chain network design under uncertainty. European Journal of Operational Research, 167(1): 96-115. https://doi.org/10.1016/j.ejor.2004.01.046

[13] Goicoechea, A., Hansen, D.R., Duckstein, L. (1982). Multiobjective Decision Analysis with Engineering and Business Applications. John Wiley \& Sons Incorporated.

[14] Charnes, A., Cooper, W.W. (1959). Chance-constrained programming. Management Science, 6(1): 73-79. https://doi.org/10.1287/mnsc.6.1.73

[15] Dantzig, G.B., Infanger, G. (1993). Multi-stage stochastic linear programs for portfolio optimization. Annals of Operations Research, 45(1): 59-76. https://doi.org/10.1007/BF02282041

[16] Abbas, M., Bellahcene, F. (2006). Cutting plane method for multiple objective stochastic integer linear programming. European Journal of Operational Research, 168(3): 967-984. https://doi.org/10.1016/j.ejor.2002.11.006

[17] Goh, M., Lim, J.Y., Meng, F. (2007). A stochastic model for risk management in global supply chain networks. European Journal of Operational Research, 182(1): 164173. https://doi.org/10.1016/j.ejor.2006.08.028

[18] Azaron, A., Brown, K.N., Tarim, S.A., Modarres, M. (2008). A multi-objective stochastic programming approach for supply chain design considering risk. International Journal of Production Economics, 116(1): 129-138. https://doi.org/10.1016/j.ijpe.2008.08.002

[19] Sakawa, M., Matsui, T. (2012). An interactive fuzzy satisficing method for multiobjective stochastic integer programming with simple recourse. https://doi.org/10.4236/am.2012.330180

[20] Han, Y.C., Huang, G.H., Li, C.H. (2008). An intervalparameter multi-stage stochastic chance-constrained mixed integer programming model for inter-basin water resources management systems under uncertainty. In 2008 Fifth International Conference on Fuzzy Systems and Knowledge Discovery, 5: 146-153. https://doi.org/10.1109/FSKD.2008.465

[21] Körpeoğlu, E., Yaman, H., Aktürk, M.S. (2011). A multistage stochastic programming approach in master production scheduling. European Journal of Operational Research, 213(1): 166-179. https://doi.org/10.1016/j.ejor.2011.02.032

[22] Birge, J.R., Louveaux, F. (2011). Introduction to stochastic programming. Springer Science \& Business Media.

[23] Wang, S., Watada, J. (2011). Two-stage fuzzy stochastic programming with Value-at-Risk criteria. Applied Soft Computing, 11(1): 1044-1056. https://doi.org/10.1016/j.asoc.2010.02.004

[24] Barik, S.K., Biswal, M.P., Chakravarty, D. (2012). Multiobjective two-stage stochastic programming problems with interval discrete random variables. Advances in Operations Research. https://doi.org/10.1155/2012/279181

[25] Abdelaziz, F.B. (2012). Solution approaches for the multiobjective stochastic programming. European Journal of Operational Research, 216(1): 1-16. https://doi.org/10.1016/j.ejor.2011.03.033 
[26] Gutjahr, W.J., Pichler, A. (2016). Stochastic multiobjective optimization: a survey on non-scalarizing methods. Annals of Operations Research, 236(2): 475499. https://doi.org/10.1007/s10479-013-1369-5

[27] Housh, M., Ostfeld, A., Shamir, U. (2013). Limited multi-stage stochastic programming for managing water supply systems. Environmental Modelling \& Software, 41: 53-64. https://doi.org/10.1016/j.envsoft.2012.11.006

[28] Kumar, P., Dutta, D. (2015). Multi-objective linear fractional inventory model of multi-products with pricedependant demand rate in fuzzy environment. International Journal of Mathematics in Operational Research, $7(5)$ : $547-565$ https://doi.org/10.1504/IJMOR.2015.071280

[29] Kilıç, Y.E., Tuzkaya, U.R. (2015). A two-stage stochastic mixed-integer programming approach to physical distribution network design. International Journal of Production Research, 53(4): 1291-1306. https://doi.org/10.1080/00207543.2014.957871

[30] Dutta, D., Kumar, P. (2015). A partial backlogging inventory model for deteriorating items with timevarying demand and holding cost. International Journal of Mathematics in Operational Research, 7(3): 281-296. https://doi.org/10.1504/IJMOR.2015.069144

[31] Yu, H., Solvang, W.D. (2016). A stochastic programming approach with improved multi-criteria scenario-based solution method for sustainable reverse logistics design of waste electrical and electronic equipment (WEEE). Sustainability, 8(12): 1331. https://doi.org/10.3390/su8121331

[32] Khalifa, H.A., Elgendi, E.A., Ebraheim, A.H.N. (2018). A fuzzy programming approach to solve stochastic multi-objective quadratic programming problems. In Science and Information Conference, pp. 262-271. https://doi.org/10.1007/978-3-030-01174-1_20

[33] Yu, H., Solvang, W.D. (2020). A fuzzy-stochastic multiobjective model for sustainable planning of a closed-loop supply chain considering mixed uncertainty and network flexibility. Journal of Cleaner Production, 266: 121702. https://doi.org/10.1016/j.jclepro.2020.121702

[34] Caglayan, N., Satoglu, S.I. (2021). Multi-objective twostage stochastic programming model for a proposed casualty transportation system in large-scale disasters: A case study. Mathematics, 9(4): 316. https://doi.org/10.3390/math9040316

[35] Mohamadi, A., Yaghoubi, S. (2017). A bi-objective stochastic model for emergency medical services network design with backup services for disasters under disruptions: An earthquake case study. International Journal of Disaster Risk Reduction, 23: 204-217. https://doi.org/10.1016/j.ijdrr.2017.05.003

[36] Restrepo, M.I., Gendron, B., Rousseau, L.M. (2017). A two-stage stochastic programming approach for multiactivity tour scheduling. European Journal of Operational Research, 262(2): 620-635 https://doi.org/10.1016/j.ejor.2017.04.055

[37] Floyd, M.K., Barker, K., Rocco, C.M., Whitman, M.G.
(2017). A multi-criteria decision analysis technique for stochastic task criticality in project management. Engineering Management Journal, 29(3): 165-178. https://doi.org/10.1080/10429247.2017.1340038

[38] Farrokh, M., Azar, A., Jandaghi, G., Ahmadi, E. (2018). A novel robust fuzzy stochastic programming for closed loop supply chain network design under hybrid uncertainty. Fuzzy Sets and Systems, 341: 69-91. https://doi.org/10.1016/j.fss.2017.03.019

[39] Rahmanniyay, F., Yu, A.J. (2019). A multi-objective stochastic programming model for project-oriented human-resource management optimization. International Journal of Management Science and Engineering Management, 14(4): 231-239. https://doi.org/10.1080/17509653.2018.1534220

[40] Ahmadi, S., Amin, S.H. (2019). An integrated chanceconstrained stochastic model for a mobile phone closedloop supply chain network with supplier selection. Journal of Cleaner Production, 226: 988-1003. https://doi.org/10.1016/j.jclepro.2019.04.132

[41] Khalifa, H.A., Kumar, P. (2020). Multi-objective optimization for solving cooperative continuous static games using Karush-Kuhn-Tucker conditions. International Journal of Operations Research. https://doi.org/10.1504/IJOR.2020.10031969.

[42] Waliv, R.H., Mishra, U., Garg, H., Umap, H.P. (2020). A nonlinear programming approach to solve the stochastic multi-objective inventory model using the uncertain information. Arabian Journal for Science and Engineering, 45: 6963-6973. https://doi.org/10.1007/s13369-020-04618-z

[43] Yu, C.S., Li, H.L. (2000). A robust optimization model for stochastic logistic problems. International Journal of Production Economics, 64(1-3): 385-397. https://doi.org/10.1016/S0925-5273(99)00074-2

[44] Yenice, Z.D., Samanlioglu, F. (2020). A multi-objective stochastic model for an earthquake relief network. Journal of Advanced Transportation, 2020: 1910632. https://doi.org/10.1155/2020/1910632

[45] Khalifa, H.A.E.W., Kumar, P., Alharbi, M.G. (2021). On characterizing solution for multi-objective fractional two-stage solid transportation problem under fuzzy environment. Journal of Intelligent Systems, 30(1): 620635. https://doi.org/10.1515/jisys-2020-0095

\section{NOMENCLATURE}

$\begin{array}{ll}\mu & \text { Mean } \\ \mathrm{V} & \text { Variance } \\ \sigma_{\mathrm{ij}, \mathrm{rl}}^{2} & \text { Covariance } \\ \mathrm{z} & \text { Standard normal variable } \\ \phi(\mathrm{z}) & \text { Cumulative density function of } \mathrm{z} \\ \Psi_{\delta_{\mathrm{i}}} & \text { Standard normal variable value } \\ \mathrm{Z}_{\mathrm{k}}^{\mathrm{L}} & \text { Lower bounds for each objective function } \\ \mathrm{Z}_{\mathrm{k}}^{\mathrm{U}} & \text { Upper bounds for each objective function }\end{array}$

